\title{
Positive solutions of discrete third-order boundary value problems with sign-changing Green's function
}

Jing Wang ${ }^{1}$ and Chenghua Gao ${ }^{1,2^{*}}$

\section{*Correspondence:}

gaokuguo@163.com

1 Department of Mathematics,

Northwest Normal University,

Lanzhou, 730070, P.R. China

${ }^{2}$ Department of Mathematics,

Lanzhou University, Lanzhou,

730000, P.R. China

\begin{abstract}
In this paper, we consider the existence of positive solutions for a discrete third-order boundary value problems, which has the sign-changing Green's function. The approach we use is the Guo-Krasnoselskii fixed point theorem in a cone.
\end{abstract}

Keywords: discrete third-order boundary value problem; sign-changing Green's function; positive solution; cone

\section{Introduction}

Let $a, b$ be two integers with $b>a$. Let us employ $[a, b]_{\mathbb{Z}}$ to denote the integer set $\{a, a+$ $1, \ldots, b\}$. For any real number $c>1,[c]$ is the integer part of $c$. In this paper, we consider the existence of a positive solution for the following discrete third-order BVP:

$$
\left\{\begin{array}{l}
\Delta^{3} u(t-1)+a(t) f(t, u(t))=0, \quad t \in[1, T-1]_{\mathbb{Z}} \\
u(0)=\Delta u(T)=\Delta^{2} u(\eta)=0
\end{array}\right.
$$

where $T>3$ is an integer, $\eta \in\left[1,\left[\frac{3 T^{2}-3 T-2}{6 T+3}\right]\right]_{\mathbb{Z}}, a:[1, T-1]_{\mathbb{Z}} \rightarrow(0,+\infty)$ and $f:[1, T-1]_{\mathbb{Z}} \times$ $[0,+\infty) \rightarrow[0,+\infty)$ is continuous.

Difference equations appear in many mathematical models in diverse fields, such as economy, biology, physics, and finance; see [1-3]. In recent years, the existence and multiplicity of positive solutions of discrete boundary value problems have received much attention from many authors and a great deal of work has been done by using classical methods such as fixed point theory [4-8], lower and upper solutions methods [9], critical point theory [10-12], etc.

Specially, Jiang et al. [6, 13], Hao [7], Gao [10], Ma et al. [14], Kong et al. [15], and Henderson et al. [16-18] considered the existence of positive solution for discrete equations by using fixed point theory in a cone.

However, in all of the above papers, in order to obtain positive solution, the Green's functions they used are positive. Now, there is a question: when the Green's function changes its sign, can we get the existence of a positive solution?

In this paper, we will consider the existence of a positive solution of (1.1). It will be shown that the Green's function of (1.1) changes its sign in Section 2. 
Finally, it must be mentioned that there are some excellent results on the existence of the positive solutions of BVPs for ordinary differential equations when the Green's functions change their signs; see Sun et al. [19-21] and Palamides et al. [22, 23] and references therein.

Our main tool is the following well-known Guo-Krasnoselskii fixed point theorem.

Theorem 1.1 [24] Let E be a Banach space and $K$ a cone in E. Assume that $\Omega_{1}$ and $\Omega_{2}$ are bounded open subsets of $E$ such that $0 \in \Omega_{1}, \bar{\Omega}_{1} \subset \Omega_{2}$, and $A: K \cap\left(\bar{\Omega}_{2} \backslash \Omega_{1}\right) \rightarrow K$ is a completely continuous operator such that either

(i) $\|A u\| \leq\|u\|$ for $u \in K \cap \partial \Omega_{1}$ and $\|A u\| \geq\|u\|$ for $u \in K \cap \partial \Omega_{2}$, or

(ii) $\|A u\| \geq\|u\|$ for $u \in K \cap \partial \Omega_{1}$ and $\|A u\| \leq\|u\|$ for $u \in K \cap \partial \Omega_{2}$.

Then $A$ has at least one fixed point in $K \cap\left(\bar{\Omega}_{2} \backslash \Omega_{1}\right)$.

The rest of this paper is arranged as follows. In Section 2, we will show the expression and some properties of the Green's function of (1.1). Specially, we will show that the Green's function changes its sign. Moreover, we will give some other preliminaries. In Section 3, we will demonstrate our main result and prove it.

\section{Preliminaries}

First, let us consider the following linear problem:

$$
\left\{\begin{array}{l}
\Delta^{3} u(t-1)+y(t)=0, \quad t \in[1, T-1]_{\mathbb{Z}} \\
u(0)=\Delta u(T)=\Delta^{2} u(\eta)=0
\end{array}\right.
$$

We will convert (2.1) to the equivalent summation equation. To get it, let us define the Green's function $G(t, s)$ as follows.

If $s>\eta$, then

$$
G(t, s)= \begin{cases}t(T-s), & t-2<s \\ \frac{(2 T+1) t-t^{2}-s^{2}-s}{2}, & s \leq t-2 .\end{cases}
$$

If $s \leq \eta$, then

$$
G(t, s)= \begin{cases}\frac{t^{2}-(1+2 s) t}{2}, & t-2<s \\ \frac{-s^{2}-s}{2}, & s \leq t-2\end{cases}
$$

Now, we get the following lemma.

Lemma 2.1 The problem (2.1) has a unique solution

$$
u(t)=\sum_{s=1}^{T-1} G(t, s) y(s),
$$

where $G(t, s)$ is defined as (2.2) and (2.3).

Proof Summing from $s=1$ to $s=t-1$ at both sides of (2.1), we get

$$
\Delta^{2} u(t-1)=\Delta^{2} u(0)-\sum_{s=1}^{t-1} y(s) .
$$


Repeating the above process, we obtain

$$
\Delta u(t-1)=\Delta u(0)+(t-1) \Delta^{2} u(0)-\sum_{s=1}^{t-2}(t-s-1) y(s)
$$

Summing from $s=1$ to $s=t$ at both sides of the above equation, we have

$$
u(t)=t \Delta u(0)+\frac{t(t-1)}{2} \Delta^{2} u(0)-\sum_{s=1}^{t-2} \frac{(t-s)(t-s-1)}{2} y(s)
$$

By using the boundary condition $u(0)=\Delta u(T)=\Delta^{2} u(\eta)=0$, we get

$$
\left\{\begin{array}{l}
\Delta^{2} u(\eta)=\Delta^{2} u(0)-\sum_{s=1}^{\eta} y(s)=0 \\
\Delta u(T)=\Delta u(0)+T \Delta^{2} u(0)-\sum_{s=1}^{T-1}(T-s) y(s)=0
\end{array}\right.
$$

Therefore,

$$
u(t)=-T t \sum_{s=1}^{\eta} y(s)+t \sum_{s=1}^{T-1}(T-s) y(s)+\frac{t(t-1)}{2} \sum_{s=1}^{\eta} y(s)-\sum_{s=1}^{t-2} \frac{(t-s)(t-s-1)}{2} y(s)
$$

which implies (2.4) holds.

Now, we can give some properties of $G(t, s)$.

If $s>\eta$, then

$$
\Delta_{t} G(t, s)= \begin{cases}T-s, & t-2<s \\ T-t, & s \leq t-2\end{cases}
$$

If $s \leq \eta$, then

$$
\Delta_{t} G(t, s)= \begin{cases}t-s, & t-2<s \\ 0, & s \leq t-2\end{cases}
$$

Thus, if $\eta<s \leq T-1$, then $G(t, s) \geq 0$ and $\Delta_{t} G(t, s) \geq 0$ for $t \in[0, T]_{\mathbb{Z}}$. We have

$$
\max _{t \in[0, T+1]_{\mathbb{Z}}}|G(t, s)|=G(T+1, s)= \begin{cases}(T+1)(T-s) \leq 2 T(T-\eta), & t-2<s \\ \frac{(T-s)(T+s+1)}{2} \leq 2 T(T-\eta), & s \leq t-2 .\end{cases}
$$

If $1 \leq s \leq \eta$, then $G(t, s) \leq 0$ and $\Delta_{t} G(t, s)>0$ for $t \in[s+1, s+2]_{\mathbb{Z}}, \Delta_{t} G(t, s) \leq 0$ for $t \in[0, s]_{\mathbb{Z}}$. We have

$$
\max _{t \in[0, T+1]_{\mathbb{Z}}}|G(t, s)|=-\min _{t \in[0, T+1]_{\mathbb{Z}}} G(t, s)= \begin{cases}0, & t-2<s \\ \frac{s^{2}+s}{2} \leq \eta^{2}+\eta, & s \leq t-2 .\end{cases}
$$

Since $\eta \in\left[1,\left[\frac{3 T^{2}-3 T-2}{6 T+3}\right]\right]_{\mathbb{Z}}$ and $T>3$, we can obtain

$$
\begin{aligned}
\max _{t \in[0, T+1]_{\mathbb{Z}}}|G(t, s)| & \leq \max \left\{2 T(T-\eta), \eta^{2}+\eta\right\} \\
& =2 T(T-\eta), \quad(t, s) \in[0, T+1]_{\mathbb{Z}} \times[1, T-1]_{\mathbb{Z}} .
\end{aligned}
$$


Remark Let us give some reasons for why $\eta \in\left[1,\left[\frac{3 T^{2}-3 T-2}{6 T+3}\right]\right]_{\mathbb{Z}}$. To get it, let us consider the following BVPs:

$$
\left\{\begin{array}{l}
\Delta^{3} u(t)=-1, \\
u(0)=\Delta u(T)=\Delta^{2} u(\eta)=0 .
\end{array}\right.
$$

By Lemma 2.1, we find that (2.7) has a solution $u(t)$ as follows:

$$
u(t)=-\frac{t^{3}}{6}+\frac{1+\eta}{2} t^{2}+\left(\frac{T(T-1)}{2}-T \eta-\frac{\eta}{2}-\frac{1}{3}\right) t .
$$

Now, we will show that if $\eta \in\left[1,\left[\frac{3 T^{2}-3 T-2}{6 T+3}\right]\right]_{\mathbb{Z}}$, then $u(t) \geq 0$ for $t \in[0, T+1]_{\mathbb{Z}}$.

Let $\phi(t)=t^{2}-3(1+\eta) t-3 T(T-1)+3(2 T+1) \eta+2$. It is obvious that $u(t) \geq 0$ equals $\phi(t) \leq 0$. Since $\Delta \phi(t)=2 t-3 \eta-2$, we get $\Delta \phi(t) \geq 0$ for $t \geq \frac{3 \eta}{2}+1$ and $\Delta \phi(t) \leq 0$ for $t \leq$ $\frac{3 \eta}{2}+1$. Due to $\frac{2 T}{3}>\frac{3 T^{2}-3 T-2}{6 T+3}$, we get $\frac{3 \eta}{2}+1<T+1$. Therefore, $\Delta \phi(t) \geq 0$ for $t \in\left[\left[\frac{3 \eta}{2}+1\right], T\right]_{\mathbb{Z}}$ and $\Delta \phi(t) \leq 0$ for $t \in\left[0,\left[\frac{3 \eta}{2}+1\right]\right]_{\mathbb{Z}}$, i.e., $\phi(t)$ is increasing on $\left[\left[\frac{3 \eta}{2}+1\right], T+1\right]_{\mathbb{Z}}$ and $\phi(t)$ is decreasing on $\left[0,\left[\frac{3 \eta}{2}+1\right]_{\mathbb{Z}}\right.$. Consequently, if $\phi(0) \leq 0$ and $\phi(T+1) \leq 0$, then $\phi(t) \leq 0$ for $t \in[0, T+1]_{\mathbb{Z}}$. By the direct computation, $\phi(0) \leq 0$ for $\eta \in\left[1,\left[\frac{3 T^{2}-3 T-2}{6 T+3}\right]\right]_{\mathbb{Z}}$ and $\phi(T+$ 1) $\leq 0$ for $\eta \in\left[1,\left[\frac{2 T^{2}-2 T}{3 T}\right]\right]_{\mathbb{Z}}$. Combining with the fact $\frac{3 T^{2}-3 T-2}{6 T+3}<\frac{2 T^{2}-2 T}{3 T}<\frac{2 T}{3}$, we get $\eta \in$ $\left[1,\left[\frac{3 T^{2}-3 T-2}{6 T+3}\right]\right]_{\mathbb{Z}}$.

Now, let us give some notations.

Let $E=\left\{u:[0, T+1]_{\mathbb{Z}} \rightarrow \mathbb{R}\right\}$. Then $E$ is a Banach space under the norm $\|u\|=$ $\max _{t \in[0, T+1]_{\mathbb{Z}}}|u(t)|$. Let

$$
K_{0}=\left\{y \in E: y(t) \geq 0, \Delta y(t) \geq 0, t \in[0, T]_{\mathbb{Z}} \text { and } \Delta^{2} y(t-1) \leq 0, t \in[\eta+1, T]_{\mathbb{Z}}\right\} .
$$

Then $K_{0}$ is a cone in $E$.

Lemma 2.2 Assume $y \in E, y(t) \geq 0$ for $t \in[0, T+1]_{\mathbb{Z}}$ and $\Delta y(t) \geq 0$ for $t \in[0, T]_{\mathbb{Z}}$. Then the unique solution $u(t)$ of (2.1) belongs to $K_{0}$, where $u(t)$ is defined as (2.4). Moreover, $u(t)$ is concave on $[\eta+1, T+1]_{\mathbb{Z}}$.

Proof The following proof will be divided into two cases.

Case I. For $0 \leq t-2<\eta$, we have

$$
u(t)=-\sum_{s=1}^{t-2} \frac{s^{2}+s}{2} y(s)+\sum_{s=t-1}^{\eta} \frac{t^{2}-(1+2 s) t}{2} y(s)+\sum_{s=\eta+1}^{T-1} t(T-s) y(s) .
$$

Since $\eta \in\left[1,\left[\frac{3 T^{2}-3 T-2}{6 T+3}\right]\right]_{\mathbb{Z}}$, we get

$$
\begin{aligned}
\Delta u(t)= & -\frac{t^{2}-t}{2} y(t-1)+\sum_{s=t}^{\eta} \frac{(t+1)^{2}-(1+2 s)(t+1)}{2} y(s) \\
& -\sum_{s=t-1}^{\eta} \frac{t^{2}-(1+2 s) t}{2} y(s)+\sum_{s=\eta+1}^{T-1}(T-s) y(s)
\end{aligned}
$$




$$
\begin{aligned}
& =-\frac{t^{2}-t}{2} y(t-1)+\sum_{s=t}^{\eta}(t-s) y(s)-\frac{-t^{2}+t}{2} y(t-1)+\sum_{s=\eta+1}^{T-1}(T-s) y(s) \\
& =\sum_{s=t}^{\eta}(t-s) y(s)+\sum_{s=\eta+1}^{T-1}(T-s) y(s) \\
& =\sum_{s=t}^{\eta}(t-s) y(s)+\sum_{s=\eta}^{T-1}(T-s) y(s)-(T-\eta) y(\eta) \\
& \geq y(\eta) \sum_{s=t}^{\eta}(t-s)+y(\eta) \sum_{s=\eta}^{T-1}(T-s)-(T-\eta) y(\eta) \\
& =y(\eta)(T-t)\left(-\eta+\frac{T+t}{2}-\frac{1}{2}\right) \geq 0 .
\end{aligned}
$$

Case II. For $\eta \leq t-2 \leq T-1$, we have

$$
u(t)=-\sum_{s=1}^{\eta} \frac{s^{2}+s}{2} y(s)+\sum_{s=\eta+1}^{t-2} \frac{(2 T+1) t-t^{2}-s^{2}-s}{2} y(s)+\sum_{s=t-1}^{T-1} t(T-s) y(s) .
$$

Furthermore, we get

$$
\begin{aligned}
\Delta u(t)= & \sum_{s=\eta+1}^{t-1} \frac{(2 T+1)(t+1)-(t+1)^{2}-s^{2}-s}{2} y(s) \\
& -\sum_{s=\eta+1}^{t-2} \frac{(2 T+1) t-t^{2}-s^{2}-s}{2} y(s) \\
& +\sum_{s=t}^{T-1}(t+1)(T-s) y(s)-\sum_{s=t-1}^{T} t(T-s) y(s) \\
= & \sum_{s=\eta+1}^{t-1}(T-t) y(s)+\sum_{s=t}^{T-1}(T-s) y(s) \geq 0
\end{aligned}
$$

and

$$
\Delta^{2} u(t-1)=-\sum_{s=\eta+1}^{t-1} y(s) \leq 0 .
$$

Since $\Delta u(t) \geq 0$ for $t \in[0, T]_{\mathbb{Z}}$, we have $u(t) \geq 0$ for $t \in[0, T+1]_{\mathbb{Z}}$. So, $u \in K_{0}$. Due to $\Delta^{2} u(\eta)=0$, we have $\Delta^{2} u(t-1) \leq 0$ for $t \in[\eta+1, T]_{\mathbb{Z}}$, i.e., $u(t)$ is concave on $[\eta+1, T+1]_{\mathbb{Z}}$.

Lemma 2.3 Assume $y \in E, y(t) \geq 0$ for $t \in[0, T+1]_{\mathbb{Z}}, \Delta y(t) \geq 0$ for $t \in[0, T]_{\mathbb{Z}}$, and $u$ is the solution of (2.1). Then $u$ satisfies

$$
\min _{t \in[\theta, T+1-\theta]_{\mathbb{Z}}} u(t) \geq \theta^{*}\|u\|
$$

where $\theta^{*}=\frac{\theta-\eta-1}{T-\eta}$ and $\theta \in\left[\eta+1,\left[\frac{3 T^{2}+3 T+1}{6 T+3}\right]\right]_{\mathbb{Z}}$. 
Proof From Lemma 2.2, we know that $u$ is concave on $t \in[\eta+1, T+1]_{\mathbb{Z}}$. Therefore,

$$
\frac{u(T+1)-u(\eta+1)}{T-\eta} \leq \frac{u(t)-u(\eta+1)}{t-\eta-1}, \quad t \in[\eta+2, T+1]_{\mathbb{Z}}
$$

Finally, by direct computation, we get

$$
u(t) \geq \frac{t-\eta-1}{T-\eta} u(T+1)=\frac{t-\eta-1}{T-\eta}\|u\| .
$$

That is,

$$
\min _{t \in[\theta, T+1-\theta]_{\mathbb{Z}}} u(t)=u(\theta) \geq \frac{\theta-\eta-1}{T-\eta}\|u\|=\theta^{*}\|u\| .
$$

Since $\frac{3 T^{2}+3 T+1}{6 T+3} \leq \frac{T+1}{2}$, we get $\theta \leq T+1-\theta$, and the set $[\theta, T+1-\theta]$ is well defined.

\section{Main results}

In this section, we conclude the existence of positive solution of (1.1). To get it, we assume that:

(H1) $f:[1, T-1]_{\mathbb{Z}} \times[0,+\infty) \rightarrow[0, \infty)$ is continuous and the mapping $u \mapsto f(t, u)$ is nondecreasing for each $t \in[1, T-1]_{\mathbb{Z}}$;

(H2) $a:[1, T-1]_{\mathbb{Z}} \rightarrow(0,+\infty)$ is increasing.

Define the cone

$$
K=\left\{u \in K_{0}: u(0)=0, \min _{t \in[\theta, T+1-\theta]_{\mathbb{Z}}} u(t) \geq \theta^{*}\|u\|\right\}
$$

and the operator $A: K \rightarrow K$ by

$$
A u(t)=\sum_{s=1}^{T-1} G(t, s) a(s) f(s, u(s)) .
$$

Obviously, if $u$ is fixed point of $A$ in $K$, then $u$ is positive and increasing solution of the BVP (1.1). From Lemma 2.2 and Lemma 2.3 , we know that $A: K \rightarrow K$ is completely continuous.

Set

$$
B=2 T(T-\eta) \sum_{s=1}^{T-1} a(s), \quad D=(\theta-1)(T-\theta+1) \sum_{s=\theta}^{T+1-\theta} a(s) .
$$

Theorem 3.1 Assume that (H1) and (H2) hold. If there exist two positive constants $r$ and $R$ with $r \neq R$ such that

(A1) $f(t, x) \leq \frac{r}{B}$ for $(t, x) \in[1, T-1]_{\mathbb{Z}} \times[0, r]$,

(A2) $f(t, x) \geq \frac{R}{D}$ for $(t, x) \in[1, T-1]_{\mathbb{Z}} \times\left[\theta^{*} R, R\right]$,

then the BVP (1.1) has a positive and increasing solution $u$ satisfying $\min \{r, R\} \leq\|u\| \leq$ $\max \{r, R\}$. Moreover, the obtained solution $u(t)$ is concave on $[\eta+1, T+1]_{\mathbb{Z}}$.

Proof Firstly, we deal with the case $r<R$. Let

$$
\Omega_{1}=\{u \in E:\|u\|<r\}, \quad \Omega_{2}=\{u \in E:\|u\|<R\} .
$$


For any $u \in K \cap \partial \Omega_{1}$, from (2.6), the assumption (A1) implies that

$$
\begin{aligned}
\|A u\| & =\max _{t \in[0, T+1]_{\mathbb{Z}}}\left|\sum_{s=1}^{T-1} G(t, s) a(s) f(s, u(s))\right| \\
& \leq 2 T(T-\eta) \sum_{s=1}^{T-1} a(s) f(s, u(s)) \\
& \leq 2 T(T-\eta) \sum_{s=1}^{T-1} a(s) \frac{r}{B} \\
& =r=\|u\| .
\end{aligned}
$$

This shows that $\|A u\| \leq\|u\|, u \in K \cap \partial \Omega_{1}$.

Similarly, for any $u \in K \cap \partial \Omega_{2}$, we get $\theta^{*} R \leq u(s) \leq R$ for $s \in[\theta, T+1-\theta]_{\mathbb{Z}}$ by Lemma 2.3. Since the function $G(t, s)$ is positive and increasing for $\eta<s \leq T-1$, it follows from the assumption (A2) that

$$
\begin{aligned}
\|A u\| & =\max _{t \in[0, T+1]_{\mathbb{Z}}}\left|\sum_{s=1}^{T-1} G(t, s) a(s) f(s, u(s))\right| \\
& =\max _{t \in[0, T+1]_{\mathbb{Z}}} \sum_{s=1}^{T-1} G(t, s) a(s) f(s, u(s)) \\
& \geq \max _{t \in[\theta, T+1-\theta]_{\mathbb{Z}}} \sum_{s=\theta}^{T+1-\theta} G(t, s) a(s) f(s, u(s)) \\
& =\sum_{s=\theta}^{T+1-\theta} G(T+1-\theta, s) a(s) f(s, u(s)) \\
& =\sum_{s=\theta}^{T+1-\theta}\left(T^{2}+T+\theta-\theta^{2}-2-s^{2}-s\right) a(s) f(s, u(s)) \\
& \geq \sum_{s=\theta}^{T+1-\theta}\left(T \theta-\theta^{2}-T+2 \theta-1\right) a(s) f(s, u(s)) \\
& \geq(\theta-1)(T-\theta+1) \sum_{s=\theta}^{T+1-\theta} a(s) \frac{R}{D}=R=\|u\| .
\end{aligned}
$$

This indicates that $\|A u\| \geq\|u\|, u \in K \cap \partial \Omega_{2}$.

Therefore, $A$ has a fixed point $u \in K \cap\left(\bar{\Omega}_{2} \backslash \Omega_{1}\right)$ from Theorem 1.1, which is a positive and increasing solution of the BVP (1.1) with $r \leq\|u\| \leq R$. Moreover, we know the obtained solution $u$ is concave on $[\eta+1, T+1]_{\mathbb{Z}}$ from the proof of Lemma 2.2.

Secondly, we deal with the case $r>R$. Let

$$
\Omega_{1}=\{u \in E:\|u\|<R\}, \quad \Omega_{2}=\{u \in E:\|u\|<r\} .
$$

Then, for each $u \in K \cap \partial \Omega_{1}$, by Lemma 2.3, we obtain

$$
\min _{t \in[\theta, T+1-\theta]_{\mathbb{Z}}} u(t)=u(\theta) \geq \theta^{*}\|u\|=\theta^{*} R
$$


The assumption (A2) gives

$$
\begin{aligned}
\|A u\| & =\max _{t \in[0, T+1]_{\mathbb{Z}}}\left|\sum_{s=1}^{T-1} G(t, s) a(s) f(s, u(s))\right| \\
& =\max _{t \in[0, T+1]_{\mathbb{Z}}} \sum_{s=1}^{T-1} G(t, s) a(s) f(s, u(s)) \\
& \geq \max _{t \in[\theta, T+1-\theta]_{\mathbb{Z}}} \sum_{s=\theta}^{T+1-\theta} G(t, s) a(s) f(s, u(s)) \\
& =\sum_{s=\theta}^{T+1-\theta} G(T+1-\theta, s) a(s) f(s, u(s)) \\
& \geq(\theta-1)(T-\theta+1) \sum_{s=\theta}^{T+1-\theta} a(s) \frac{R}{D}=R=\|u\| .
\end{aligned}
$$

If $u \in K \cap \partial \Omega_{2}$, then $0 \leq u(s) \leq r, 1 \leq s \leq T-1$. So, the assumption (A1) yields

$$
\begin{aligned}
\|A u\| & =\max _{t \in[0, T+1]_{\mathbb{Z}}} \sum_{s=1}^{T-1} G(t, s) a(s) f(s, u(s)) \\
& \leq 2 T(T-\eta) \sum_{s=1}^{T-1} a(s) \frac{r}{B}=r=\|u\| .
\end{aligned}
$$

Therefore, it is clear that the result holds.

Corollary 3.1 Suppose that $(\mathrm{H} 1)$ and $(\mathrm{H} 2)$ hold. Iff satisfies

(A3) $\lim _{x \rightarrow 0^{+}} \max _{t \in[1, T-1]_{\mathbb{Z}}} \frac{f(t, x)}{x}=0$ and $\lim _{x \rightarrow+\infty} \min _{t \in[1, T-1]_{\mathbb{Z}}} \frac{f(t, x)}{x}=+\infty$, or

(A4) $\lim _{x \rightarrow 0^{+}} \min _{t \in[1, T-1]_{\mathbb{Z}}} \frac{f(t, x)}{x}=+\infty$ and $\lim _{x \rightarrow+\infty} \max _{t \in[1, T-1]_{\mathbb{Z}}} \frac{f(t, x)}{x}=0$,

then $B V P(1.1)$ has a positive and increasing solution $u$, which is concave on $[\eta+1, T+1]_{\mathbb{Z}}$.

Proof Superlinear case. Since $\lim _{x \rightarrow 0^{+}} \max _{t \in[1, T-1]_{\mathbb{Z}}} \frac{f(t, x)}{x}=0$, there exists a constant $r_{1}>0$ so that

$$
\frac{f(t, x)}{x} \leq \frac{r_{1}}{B}, \quad(t, x) \in[1, T-1]_{\mathbb{Z}} \times\left[0, r_{1}\right] .
$$

Similarly, since $\lim _{x \rightarrow+\infty} \min _{t \in[1, T-1]_{\mathbb{Z}}} \frac{f(t, x)}{x}=+\infty$, there exists a constant $R_{1}>r_{1}$ so that

$$
f(t, x) \geq \frac{x}{\theta^{*} D} \geq \frac{\theta^{*} R_{1}}{\theta^{*} D}=\frac{R_{1}}{D}, \quad(t, x) \in[1, T-1]_{\mathbb{Z}} \times\left[\theta^{*} R_{1}, R_{1}\right] .
$$

Hence, by Theorem 3.1, we get the desired result.

Sublinear case. Firstly, we let

$$
\Omega_{1}=\left\{u \in E:\|u\|<r_{1}\right\}
$$

For $u \in K \cap \partial \Omega_{1}$, we get $\theta^{*} r_{1} \leq u(s) \leq r_{1}$ for $s \in[\theta, T+1-\theta]_{\mathbb{Z}}$. 
Since $\lim _{x \rightarrow 0^{+}} \min _{t \in[1, T-1]_{\mathbb{Z}}} \frac{f(t, x)}{x}=+\infty$, there exists a constant $r_{1}<R_{1}$ so that

$$
f(s, u(s)) \geq \frac{u(s)}{D \theta^{*}} \geq \frac{\theta^{*} r_{1}}{D \theta^{*}}=\frac{r_{1}}{D}, \quad(s, u(s)) \in[1, T-1]_{\mathbb{Z}} \times\left[\theta^{*} r_{1}, r_{1}\right] .
$$

Consequently, for $u \in K \cap \partial \Omega_{1}$, we get

$$
\begin{aligned}
\|A u\| & =\max _{t \in[0, T+1]_{\mathbb{Z}}}\left|\sum_{s=1}^{T-1} G(t, s) a(s) f(s, u(s))\right| \\
& =\max _{t \in[0, T+1]_{\mathbb{Z}}} \sum_{s=1}^{T-1} G(t, s) a(s) f(s, u(s)) \\
& \geq \max _{t \in[\theta, T+1-\theta]_{\mathbb{Z}}} \sum_{s=\theta}^{T+1-\theta} G(t, s) a(s) f(s, u(s)) \\
& =\sum_{s=\theta}^{T+1-\theta} G(T+1-\theta, s) a(s) f(s, u(s)) \\
& \geq(\theta-1)(T-\theta+1) \sum_{s=\theta}^{T+1-\theta} a(s) \frac{r_{1}}{D}=r_{1}=\|u\| .
\end{aligned}
$$

Now, we consider this case $\lim _{x \rightarrow+\infty} \max _{t \in[1, T-1]_{\mathbb{Z}}} \frac{f(t, x)}{x}=0$.

Let

$$
\Omega_{2}=\left\{u \in E:\|u\|<R_{1}\right\}
$$

Case I. $f$ is bounded. Then there exists a constant $M>0$ so that $f(t, u) \leq M$ for $(t, u) \in$ $[1, T-1]_{\mathbb{Z}} \times[0,+\infty)$. Choosing constant $R_{1} \geq B M$, we have

$$
\begin{aligned}
\|A u\| & =\max _{t \in[0, T+1]_{\mathbb{Z}}}\left|\sum_{s=1}^{T-1} G(t, s) a(s) f(s, u(s))\right| \\
& \leq 2 T(T-\eta) \sum_{s=1}^{T-1} a(s) f(s, u(s)) \\
& \leq M B \leq R_{1}=\|u\| .
\end{aligned}
$$

Therefore, $\|A u\| \leq\|u\|$ for $u \in K \cap \partial \Omega_{2}$.

Case II. $f$ is unbounded. Then we let constant $R_{1}$ be positive and large enough such that

$$
f(t, u) \leq f\left(t, R_{1}\right) \leq \frac{R_{1}}{B}, \quad(t, u) \in[1, T-1]_{\mathbb{Z}} \times\left[0, R_{1}\right]
$$

Consequently, for $u \in K \cap \partial \Omega_{2}$, we get

$$
\begin{aligned}
\|A u\| & =\max _{t \in[0, T+1]_{\mathbb{Z}}}\left|\sum_{s=1}^{T-1} G(t, s) a(s) f(s, u(s))\right| \\
& \leq 2 T(T-\eta) \sum_{s=1}^{T-1} a(s) f(s, u(s)) \leq \frac{R_{1}}{B} B=\|u\| .
\end{aligned}
$$


Therefore, by Theorem 1.1, we obtain a solution of the problem (1.1). Moreover, we know the obtained solution $u$ is concave on $[\eta+1, T+1]_{\mathbb{Z}}$ from the proof of Lemma 2.2. So, the proof of Corollary 3.1 is completed.

\section{Competing interests}

The authors declare that they have no competing interests.

Authors' contributions

All authors contributed equally to the writing of this paper. All authors read and approved the final manuscript.

\section{Acknowledgements}

The authors are very grateful to the anonymous referees for their valuable suggestions. The authors are supported by NWNU-LKQN-11-23, NSFC (11401479, 11201378), Natural Science Foundation of Gansu Province (145RJYA237), The Science Research Project for Colleges and Universities of Gansu Province (2013A-001), China Postdoctoral Science Foundation (2014M562472).

Received: 9 October 2014 Accepted: 30 January 2015 Published online: 24 February 2015

\section{References}

1. Agarwal, RP, Wong, PJY: Advanced Topics in Difference Equations. Kluwer Academic, Dordrecht (1997)

2. Agarwal, RP: Difference Equations and Inequalities: Theory, Methods and Applications. Dekker, New York (2000)

3. Liu, D, Lin, L: On the toroidal Leibniz algebras. Acta Math. Sin. Engl. Ser. 24, 227-240 (2008)

4. Atici, FM, Guseinov, GS: Positive periodic solutions for nonlinear difference equations with periodic coefficients. J. Math. Anal. Appl. 232, 166-182 (1999)

5. Henderson, J, Luca, R: On a multi-point discrete boundary value problem. J. Differ. Equ. Appl. 19, 690-699 (2013)

6. Jiang, D, Chu, J, O’Regan, D, Agarwal, RP: Positive solutions for continuous and discrete boundary value problems to the one-dimension p-Laplacian. Math. Inequal. Appl. 7, 523-534 (2004)

7. Hao, Z: Nonnegative solutions for semilinear third-order difference equation boundary value problems. Acta Math. Sci. Ser. A Chin. Ed. 21, 225-229 (2001)

8. Pang, $\mathrm{H}, \mathrm{Ge}, \mathrm{W}$ : Positive solution of second-order multi-point boundary value problems for finite difference equation with a $p$-Laplacian. J. Appl. Math. Comput. 26, 133-150 (2008)

9. Atici, FM, Cabada, A: Existence and uniqueness results for discrete second-order periodic boundary value problems Comput. Math. Appl. 45, 1417-1427 (2003)

10. Gao, C: Solutions to discrete multiparameter periodic boundary value problems involving the $p$-Laplacian via critical point theory. Acta Math. Sci. Ser. B Engl. Ed. 34, 1225-1236 (2014)

11. Yu, J, Zhu, B, Guo, Z: Positive solutions for multiparameter semipositone discrete boundary value problems via variational method. Adv. Differ. Equ. 2008, Article ID 840458 (2008)

12. Agarwal, RP, Perera, K, O'Regan, D: Multiple positive solutions of singular discrete $p$-Laplacian problems via variational methods. Adv. Differ. Equ. 2005, 93-99 (2005)

13. Yuan, C, Wen, X, Jiang, D: Existence and uniqueness of positive solution for nonlinear singular 2 nth-order continuous and discrete Lidstone boundary value problems. Acta Math. Sci. Ser. B Engl. Ed. 31, 281-291 (2011)

14. Ma, R, Gao, C, Chang, Y: Existence of solutions of a discrete fourth-order boundary value problem. Discrete Dyn. Nat. Soc. 2010, Article ID 839474 (2010)

15. Kong, L, Kong, Q, Zhang, B: Positive solutions of boundary value problems for third-order functional difference equations. Comput. Math. Appl. 44, 481-489 (2002)

16. Henderson, J, Luca, R: Existence of positive solutions for a system of second-order multi-point discrete boundary value problems. J. Differ. Equ. Appl. 19, 1889-1906 (2013)

17. Henderson, J, Luca, R: On a second-order nonlinear discrete multi-point eigenvalue problem. J. Differ. Equ. Appl. 20, 1005-1018 (2014)

18. Agarwal, RP, Henderson, J: Positive solutions and nonlinear eigenvalue problems for third-order difference equations. Comput. Math. Appl. 36, 347-355 (1998)

19. Sun, J, Zhao, J: Multiple positive solutions for a third-order three-point boundary value problem with sign-changing Green's function. Electron. J. Differ. Equ. 2012, 118 (2012)

20. Li, X, Sun, J, Kong, F: Existence of positive solution for a third-order three-point BVP with sign-changing Green's function. Electron. J. Qual. Theory Differ. Equ. 2013, 30 (2013)

21. Sun, J, Zhao, J: Iterative technique for a third-order three-point BVP with sign-changing Green's function. Electron. J. Differ. Equ. 2013, 215 (2013)

22. Palamides, AP, Smyrlis, G: Positive solutions to a singular third-order three-point BVP with an indefinitely signed Green's function. Nonlinear Anal. 68, 2104-2118 (2008)

23. Palamides, AP, Veloni, AN: A singular third-order three-point boundary value problem with nonpositive Green's function. Electron. J. Differ. Equ. 2007, 151 (2007)

24. Guo, D, Lakshmikantham, V: Nonlinear Problems in Abstract Cones. Academic Press, New York (1988) 